\title{
A VALORIZAÇÃO DA PRECEPTORIA PARA FORTALECIMENTO DA INTEGRAÇÃO ENSINO-SERVIÇO: UM ESTUDO QUALITATIVO
}

\author{
Francisco José Passos Soares1, Helga Maria Teixeira Cassiano1, Jorge Artur Peçanha de \\ Miranda Coelho1
}

1Universidade Federal de Alagoas, Brasil. francisco_passos01@hotmail.com; enfahelga@hotmail.com; jorge.coelho@famed.ufal.br

\begin{abstract}
Resumo. Introdução: O exercício da preceptoria tem sido uma realidade a partir das mudanças advindas na política governamental de formar profissionais de saúde com foco nos princípios e demandas do Sistema Único de Saúde. Após dez anos de reforma curricular houve a necessidade de identificar as fortalezas e fragilidades na efetivação da Integração Ensino-Serviço. Objetivos: Compreender as motivações para a adesão, permanência e evasão ao exercício da preceptoria. Métodos: Estudo de caso de natureza qualitativa. Foram entrevistados dezessete preceptores em Unidades Básicas de Saúde da Família sobre a experiência no exercício da preceptoria abrangendo responsabilidades institucionais, fatores determinantes à adesão, permanência ou desistência do papel de preceptor. As falas foram analisadas por meio do software Iramuteq. Resultados: A pesquisa revelou como fatores dificultadores para a adesão e permanência dos preceptores a fragilidade na gestão da integração ensino-serviço, interferência do modelo hegemônico liberal do trabalho em saúde na prática da preceptoria; precariedade dos serviços; inexistência de política de formação pedagógica e de incentivos institucionais e financeiros. Conclusões: Há a necessidade de reconhecimento oficial da função de preceptor pela academia e serviço redimensionando a importância dos estágios em saúde da família para a formação médica.
\end{abstract}

Palavras-chave: Preceptoria; Atenção Primária em Saúde; Educação; Medicina.

VALUING THE PRECEPTORY FOR STRENGTHENING EDUCATION-SERVICE INTEGRATION: A QUALITATIVE STUDY

Abstract. Introduction: The exercise of preceptorship has been a reality based on the changes resulting from the government policy of training health professionals with a focus on the principles and demands of the Unified Health System. After ten years of curricular reform there was a need to understand the strengths and weaknesses in the effectiveness Teaching-Service Integration. Objectives: To understand the motivations for adherence, permanence and evasion to the exercise of preceptorship. Methods: Qualitative case study. Seventeen preceptors were interviewed in Basic Family Health Units about the experience in the exercise of preceptorship, covering institutional responsibilities, factors determining adherence, permanence or giving up the role of preceptor. The speeches were analysed using the Iramuteq software. Results: The research revealed as factors that hinder the adherence and permanence of the preceptors the fragility in the management of the teaching-service integration, interference of the liberal hegemonic model of health work in the preceptorship practice; precariousness of services; lack of pedagogical training policy and institutional and financial incentives. Conclusions: There is a need for official recognition of the role of preceptor by the academy and service, redimensioning the importance of internships in family health for medical training.

Keywords: Preceptorship; Primary Health Care; Education; Medicine.

\section{INTRODUÇÃO}

O Sistema Único de Saúde (SUS) consolidou-se com a Constituição Federal de 1988 e foi regulamentado pela Lei no 8.080 (1999). O Decreto no 7.508 (2011) veio dispor sobre a organização relativa às ações de planejamento e de assistência à saúde, com articulação 
interfederativa, despontando o setor saúde como corresponsável pela formação de profissionais de saúde, em parceria com o setor educação.

O Art. 27 da Lei no $8.080 / 90$ prevê que os serviços públicos integrantes do SUS sejam espaços de vivência prática e de pesquisa, movimentando a dinâmica de Integração EnsinoServiço (IES). O Ministério da Saúde (MS, 2005), na perspectiva de fortalecer o SUS implementou ações para a reestruturação do modelo de atenção à saúde, elegendo a atenção básica e a formação profissional.

Nesse sentido, a formação emergiu se contrapondo a antiga construção de saberes baseados em estruturas rígidas e práticas corporativistas, advindas do modelo flexneriano, incorporadas no Brasil, exigindo um processo de mobilização interinstitucional capaz de promover a formação em saúde pautada em novos paradigmas (Cyrino, Pinto, Oliveira, \& Figueiredo, 2015).

Esforços de cunho macropolítico foram determinantes para as mudanças: em 2001 e 2014, foram propostas e atualizadas as Diretrizes Curriculares Nacionais (DCN) pelo Conselho Nacional de Educação (CNE), fortalecendo a atenção básica como cenário de formação médica (Ministério da Educação e Cultura [MEC], 2001; Ministério da Educação e Cultura [MEC], 2014 ); políticas estratégicas instrumentalizaram as mudanças na formação com o Pró- Saúde em 2005 e o Pet-Saúde em 2008 (Lima \& Rozendo, 2015; Rodrigues, Juliano, Melo, Beck, \& Prestes, 2012).

Com as DCN os cursos desenharam propostas com o compromisso ético de formar indivíduos com senso crítico sobre seu papel frente à sociedade, instigar o aluno a adquirir habilidades em comunicação, liderança, administração e gerenciamento dos serviços e da própria aprendizagem.

O MS indica os serviços públicos de saúde como lócus principal do processo ensino aprendizagem onde as ações políticas ganham materialidade plena e expressam possibilidades de criação e de apropriação de produção/reprodução no cotidiano social, e nesse aspecto a preceptoria adquire importância e valor (Pinheiro \& Luz, 2003).

A importância da IES reside, principalmente, na preocupação com o perfil e o papel do preceptor no contexto da formação médica, tendo o referencial de competência como norteador de uma identidade coletiva (Missaka \& Ribeiro, 2011).

A reordenação da formação médica sob a égide da política de educação permanente do SUS foi iniciada fortalecida com o Pro-Pet-Saúde, em 2012, caracterizando-se pela 
perspectiva interprofissional e eleição da preceptoria como estratégia essencial à efetivação da IES e alicerce à aprendizagem significativa para promover a inserção do aluno na realidade do SUS (Trajman, Assunção, Venturi, Tobias, \& Brant, 2009).

São atributos dos preceptores o compromisso com a aprendizagem, o conhecimento de seu papel como educador e a capacidade de instigar o aluno para a aprendizagem (Bentes et al., 2013; Cavalheiro \& Guimarães, 2011).

Diante da relevância da preceptoria na formação em saúde, fez-se necessário ouvir os preceptores de um curso público de medicina do nordeste, como estes se percebem na relação com o serviço, com a academia, no exercício da preceptoria. Foram verificados os determinantes da adesão do preceptor ao exercício da preceptoria, bem como da permanência e desistência.

\section{METODOLOGIA}

Estudo de caso, analítico, de natureza qualitativa, utilizando-se a entrevista semiestruturada, com sete perguntas norteadoras a respeito do exercício da preceptoria na atenção básica: 1) Como você percebe a formação médica? 2) Como você percebe os estágios na rede de atenção básica? 3) Como você percebe o compromisso e a responsabilidade da gestão municipal e federal para com a formação médica? 4) Como você percebe o papel da Universidade? 5) O que faria você desistir/retornar ao exercício da preceptoria? 6) Como você percebe a sua vivência na preceptoria? 7) O que você sugere para melhorar/adequar o exercício da preceptoria?

Participaram do estudo 17 médicos da rede de atenção básica, que atuam ou atuaram na preceptoria médica. Foram considerados como critérios de inclusão, ser médico lotado nas UBSF do $5^{\circ}$ e $7^{\circ}$ distrito sanitário de Maceió e ter atuado ou estar atuando como preceptor. O critério de exclusão adotado foi estar afastado das atividades laborais por licença ou aposentadoria, ou ainda a recusa à assinatura do TCLE.

A coleta de dados foi realizada por única pesquisadora, com entrevistas individualizadas que duraram entre trinta e oito e setenta minutos. Foram realizados contatos prévios com os participantes da pesquisa, informando-os sobre o projeto e agendando dia e hora para as entrevistas. Foi lido o TCLE e solicitadas assinatura e permissão para gravação das falas, que, transcritas, foram analisadas por meio do software IRAMUTEQ (Camargo \& Justo, 2013; Ratinaud, 2014). 
O software apoia-se em cálculos sobre a co-ocorrência de palavras em segmentos de texto, reunindo-os em classes, de acordo com sua semelhança e dessemelhança. O processo objetiva alcançá-las por meio da classificação estatística de enunciados simples do corpus considerado. No entanto, a definição final das categorias foi realizada por dois pesquisadores, independentes, considerando após leitura de todas as transcrições. A discussão foi apoiada na produção científica, nacional, recente, sobre a formação médica orientada para o SUS.

Esta pesquisa foi aprovada pelo Comitê de Ética e Pesquisa da Universidade Federal de Alagoas, com o número de certificado de apresentação para apreciação ética 49704115.8.0000.5013.

\section{RESULTADOS E DISCUSSÃO}

A análise dos resultados, constituída pelo corpus de 118 textos, apresentou um número de 1076 segmentos de texto. Destes, foram aproveitados 1064, correspondendo a $98,88 \%$ do total corpus. Considera-se tal resultado como um significativo aproveitamento do corpus, tendo em vista que foi superior a $75 \%$. A análise permitiu a identificação de três classes distintas, porém complementares: Classe 1 - Desafios à integração da gestão ensinoserviço; Classe 2 - A precariedade do trabalho; Classe 3 - A valorização da atenção básica como cenário de ensino-aprendizagem.

\subsection{Classe 1: Desafios à Integração da Gestão Ensino-Serviço}

Nas falas dos entrevistados, identificam-se aspectos objetivos e subjetivos relativos à vivência da preceptoria na rede de atenção básica de Maceió. Estes convergem e dão visibilidade aos desafios que permeiam a baixa efetividade e que contribuem para fragilidade da IES: as condições atuais de como se dá a interação, a comunicação, a corresponsabilidade das instituições e seus gestores (universidade, secretarias de saúde, coordenadores de serviços e diretores de unidade) envolvidos no apoio à preceptoria, que se expressam na indefinição de papéis e formalidade na relação academia-serviço. Os laços desta relação ainda não são suficientes para criar e assegurar condições de viabilidade ao exercício da preceptoria nas UBSF.

"Eu vejo como uma grande responsabilidade dos poderes tanto principal federal e acho que nesse aspecto nós vivemos um momento desafiador, porque assim nós estamos enfrentando muitas carências, eu acho que falta nessa relação com a gestão com o serviço uma comunicação efetiva". 
"Então, eu acho que devia haver uma interação maior da rede municipal com a federal na formação médica".

O entendimento dos entrevistados corrobora com o relato de Sisson (2009) que ao descrever sobre o desafio de conduzir as mudanças na formação dos profissionais de saúde sob a égide do princípio da integralidade, apregoa a necessidade de um repensar políticosocial, uma revisão de papéis e poderes, e construção de diálogo em todos os níveis de gestão. Ademais, aponta os setores da educação e saúde detentores do domínio legal, técnico e político para ordenar a formação de profissionais de saúde (Sisson, 2009).

Cavalheiro e Guimarães (2011) relatam que a partir das mudanças na formação de recursos humanos $(\mathrm{RH})$ para o SUS, inicia-se o movimento da IES. A academia e o serviço são desafiados em suas especificidades e potencialidades: a universidade por ser lócus privilegiado da construção do saber, reconhecer que o serviço produz conhecimento, e o serviço, por sua vez, compreender que a parceria com a academia qualifica esta produção. Segundo os relatos dos preceptores, é tímida a contribuição da universidade e do serviço ao movimento para mudanças no perfil do egresso de medicina para atender as demandas sociais. Essa tendência de reorientação do perfil com alianças para mudanças na formação é recente, em que pese o reconhecimento da necessidade de mudanças ser algo urgente. Esforços macropolíticos têm apontado estratégias para legitimar e apoiar o compromisso dos setores de educação e saúde (Pinheiro \& Luz, 2003).

Iniciativas isoladas dos setores de saúde e educação não são suficientes para responder às necessidades da formação de recursos humanos. A efetivação da integração ensino-serviço demanda medidas mais abrangentes na relação entre as duas instâncias. Diante disso, os Ministérios da Educação e Saúde, através de Portaria Interministerial de no 1.127 (2015), instituem o Contrato Organizativo de Ação Pública de Ensino-Saúde (COAPES), para fortalecer a IES no âmbito do SUS, iniciativa que não obteve sucesso.

Os preceptores apontam de forma persistente para a falta de diálogo e compromisso entre gestores do serviço e da universidade frente à preceptoria. Referindo-se à deficiência na preparação para a vivência da preceptoria, eles responsabilizam a academia. Em relação ao serviço, relatam que há pouco envolvimento no processo ensino-aprendizagem, uma vez que apenas oferecem as UBSF, sem o compromisso de verificação e adequação destes cenários.

“Na verdade há falta de compromisso da gestão municipal com a preceptoria, eles simplesmente levam o aluno para lá, mas eles não têm nenhum compromisso e essa preparação da Universidade, que eu acho seria importantíssima". 
"Eu percebo assim: que em questão tem que haver uma estruturação melhor tanto a respeito da preparação dos preceptores como o envolvimento maior da Secretaria Municipal de Saúde junto à Universidade".

As falas dos preceptores concordam com o que defendem Lins, Soares e Coelho (2016), ser o preceptor um detentor de conhecimentos, capaz de desempenhar uma pluralidade de competências. Estas condições requerem investimento para o desenvolvimento de competências pedagógicas com vistas à melhoria do processo ensino-aprendizagem.

Peduzzi, Norman, Germani, Silva e Souza (2013) e Missaka e Ribeiro (2011) situam a preceptoria como condição estratégica no atual processo de ensino-aprendizagem, fazendose necessário o apoio da gestão acadêmica bem como municipal para a oferta contínua de cursos voltados à qualificação para a docência, em uma lógica emancipatória.

Nesta pesquisa, identificou-se ainda que, apesar de não liderar o ranking de demandas, o incentivo financeiro, motiva para o exercício da preceptoria. Observa-se nas falas a crítica por não haver uma política financeira estruturante capaz de dar respostas à efetivação da preceptoria.

"A falta de incentivo, incentivo com uma bolsa, incentivo nos aspectos da responsabilidade com o aluno e gente não viu um suporte tanto da parte da Secretaria Municipal como da própria universidade. Eu acho que tinha que melhorar todo esse contexto, melhorar essa relação entre o aluno e o preceptor".

"A universidade junto à gestão municipal deveria formalizar a bolsa porque seria um estímulo para um determinado profissional se interessar em ser preceptor além de que eu acho que tem que ter uma reformulação no atendimento da unidade".

Esses relatos demonstram insatisfação com a gestão do processo de trabalho tornando evidente a necessidade de reconhecimento da adição da função pedagógica, não contratual, às atividades laborais, exigindo do médico novas responsabilidades. $A$ falta de reconhecimento e valorização, quer seja financeira e/ou do desempenho, provoca desestímulo, descrédito, afastamento, desistência da preceptoria (Bentes et al., 2013).

O poder exercido de forma verticalizada pela gestão no serviço, sobre o processo de trabalho, ignora as especificidades da preceptoria, e descuida da exigência de relações horizontalizadas para o êxito no trabalho em equipe, cooperativo e solidário (Cavalheiro \& Guimarães, 2011).

Trajman et al. (2009) consideram a redução na produção diária e melhorias na infraestrutura como mais importantes do que o incentivo financeiro.

Há entendimento pelos preceptores entrevistados de que existe a intenção por parte da academia e do serviço em estreitar o vínculo da IES, com necessidade de revisão dos compromissos e responsabilidades. 
"Eu acho que eles têm a intenção, tem que ter o compromisso tanto da gestão municipal como da federal para a formação médica."

"Espero que esse seu trabalho seja uma mudança junto à universidade e gestão municipal na preceptoria, porque elas precisam acontecer. O aluno precisa ir à unidade de atenção básica, eles precisam ter esse respaldo, aí eu acho que o seu trabalho vem melhorar tudo isso aí."

Missaka e Ribeiro (2011) afirmam que a preceptoria colabora com a construção de saber significativo para uma formação humanizada e orientada pelos princípios do SUS. Por sua vez, a academia vê-se compelida a rever interfaces entre mercado de trabalho e adequação curricular.

Lins et al. (2016) inferem que a formação dos profissionais de saúde com vistas a atender aos princípios da atenção primária em saúde (APS) exige vivência da realidade do SUS e apropriação dos conhecimentos produzidos nos campos das práticas, com abordagens pedagógicas inovadoras.

Souza (2013) descreve a preceptoria como atividade complementar à formação, garantidora da experiência com a realidade social, devendo ser instrumentalizado com as competências necessárias para o compromisso da aprendizagem mútua com o aluno.

Nesse sentido, uma política para mudança tem que ser capaz de ir além das previsões legais, das proposituras formais, das declarações de intenção, e das instâncias, para que adquira materialidade social. Para isso, deve provocar o pensamento crítico em todos os atores inclusos no processo da IES (Ceccim \& Feuerwerker, 2004).

\subsection{Classe 2: A Precariedade do Trabalho}

Esta classe expõe as falhas da gestão do ensino no trabalho em saúde, bem como seus efeitos e consequências no trabalho individual e no exercício da preceptoria, dependentes do fluxo organizacional da assistência.

Ao preceptor é imposta a responsabilidade da formação do aluno sem consideração à multiprofissionalidade e ao trabalho em equipe. Propiciar ao aluno uma vivência prática pautada em relações precárias, sem reconhecimento da integralidade assistencial, é relegar ao discente uma visão fragmentária e insuficiente do trabalho em saúde, na contramão dos ordenamentos e princípios do SUS.

\footnotetext{
"A gente consegue fazer alguma coisa antes do exame chegar ou antes mesmo do especialista me dar uma resposta, mas acho que essa rede que cerca a atenção básica precisa ser melhor estruturada”.

"Veja, vou atender os pacientes e peço o mínimo possível de exames, aí chegam aqui no CORA, não está com a internet, nem tem previsão de quando vamos marcar".
} 
Essas falas denotam preocupação com a qualidade de um ensino associado a fragmentação e insuficiência na assistência. Lins et al. (2016) afirmam que tais comprometimentos não invalidam a presença do aluno nas UBSF, uma vez que os próprios discentes consideram válida a experiência.

Segundo Trajman et al. (2009) o processo de trabalho imerso na precariedade do serviço subjuga a produção criativa das ações de saúde a uma lógica de insatisfação. Em contrapartida a essa lógica o preceptor deve despertar para a crítica sobre o processo de trabalho, e elevar sua formação profissional e ética.

Os preceptores relatam dificuldades para o trabalho em equipe: falhas na gestão política e organizacional, dos ambientes e processos de trabalho, dificultam a interação entre os profissionais.

"Porque aí você vai discutir cada caso, mas aqui a gente não tem isso, teve tempos de paciente fazer pré-natal todinho comigo e não ter um exame de sangue, isso é angustiante."

"Ficou dentro da meta dos médicos, a gente tirou uma meta plausível para que eles vejam, eles têm que ver $x$ pacientes hipertensos, diabéticos, gestantes, crianças, mas um número bem mais reduzido do que o profissional médico, até porque ele tem que acompanhar os demais profissionais da equipe".

A interprofissionalidade organizadora de um trabalho em equipe efetivo, surge como um novo princípio da APS, dificultado pelo estado atual de fragmentação do trabalho e dissociação da formação entre os distintos cursos da saúde (Peduzzi, Norman, Germani, Silva, \& Souza, 2013).

Os relatos dos participantes do estudo apontam que aspectos da precariedade da rede de atenção básica, relacionados à atividade laboral, como as exigências de cumprimento de metas de produtividade e as péssimas condições de trabalho associadas à experiência da preceptoria são, de fato, condições cruciais à desistência do papel de preceptor.

Percebe-se nas falas que as funções cumulativas de profissional e preceptor imprimem a ideia de exploração da força de trabalho, com exigências de produtividade, sem redução de metas, e sem o adicional financeiro, correspondente, induzindo ao deficiente exercício da preceptoria. A sobrecarga laboral faz o profissional temer uma avaliação negativa de sua imagem pelo aluno.

"Porque eu gostava de ser preceptora. O fator financeiro não influenciava tanto, foi mais a questão física mesmo, não tinha condição de atender o aluno aqui na unidade, se eu tivesse um local adequado para receber meus alunos aí com certeza aceitaria." 
"Se eu o deixasse atender só, eu não podia atender a mesma quantidade de pacientes, mas a mim sempre foi exigido a mesma quantidade de pacientes de quem não é preceptor, de quem não estava ali com o aluno."

Para Trajman et al. (2009) as UBSF como espaços de construção do saber e de promoção à saúde necessitam alocação de recursos suficientes para manter adequada estrutura física, condições de trabalho, fluxo organizacional e prática de saúde com perspectivas na integralidade e interdisciplinaridade, para fortalecimento do SUS.

Há preocupação por parte do preceptor em ter sua imagem profissional vinculada à realidade de precariedade na qual estão imersas as UBSF.

Sisson (2009) e Missaka e Ribeiro (2011) afirmam que a identidade profissional é adquirida na vivência do aluno no campo das práticas. É neste universo que o aluno e o preceptor põem seus conhecimentos a serviço de uma prática assistencial, a qual fortalece valores relativos ao modo de pensar e atuar, individual e/ou coletivamente. O preceptor exerce influência no discente, e isto implica desenvolver no aluno valores sociais a partir de suas experiências vivenciadas no estágio.

\subsection{Classe 3: A Valorização da Atenção Básica como Cenário de Ensino- Aprendizagem.}

Esta classe demonstra elementos positivos vivenciados pelos preceptores. Estes reconhecem que a formação na rede de atenção básica, desde o primeiro ano de graduação, vem provocando no discente o desenvolvimento gradual de segurança, autonomia, e identidade social e profissional.

Constata-se o reconhecimento da contribuição dos estágios nas UBSF para o desempenho do aluno no futuro exercício da medicina.

"Então, essa nossa formação, creio que, os nossos alunos já saem mais seguros, eles passam dois anos em prática e isso a gente tem uma fala de nossos colegas preceptores do rural, o estágio no interior".

"Eu acho que esses estágios na rede de atenção básica são necessários para o aluno de medicina, tanto o estágio inicial no $1^{\circ}$ ano de medicina como o estágio no final do curso".

Barreto, Monteiro, Magalhães, Almeida e Souza (2011) afirmam que a coprodução da autonomia é o foco de todas as relações existentes na produção do cuidar mediadas pelo ensino. A rede de atenção básica como campo potencial de práticas necessárias e o profissional como preceptor são, portanto, fundamentais para a formação em saúde. 
O preceptor reflete sobre a importância política de seu papel para a formação médica atual, demonstrando conhecimento sobre o projeto político pedagógico do curso de medicina.

\footnotetext{
“Eu acho bastante válido, importante, principalmente porque o aluno inserido na unidade de atenção básica ele vai vivenciar a realidade da medicina brasileira, e principalmente porque eu tenho uma paixão específica pelo ensino."

"Chegam as DCN e diminuem a fase de conhecimento para quatro anos, deixa dois anos finais para o internato, ou seja, onde estão os estágios, e aí um deles é o estágio na atenção básica."
}

As falas revelam a valorização da atenção básica como espaço de construção de saberes e de mudanças na formação médica. De acordo com Barreto et al. (2011) a aprendizagem na APS acontece em um cenário privilegiado para o ensino médico, pois inserida no contexto social real, com possibilidades de observar, acompanhar e identificar os determinantes sociais do processo saúde-doença.

Cavalcante, Soares e Correia (2014) sugerem que o processo ensino-aprendizagem em estágios nas UBSF provoca no discente a capacidade crítico-reflexiva sobre o processo de trabalho, com reconhecimento das limitações impostas aos profissionais e início de um processo de aquisição de atributos que conferem saber, competências, habilidades e autonomia. Para esses autores a qualidade dos cenários de ensino-aprendizagem em Maceió, nas UBSF, com foco nos atributos próprios à atenção primária, contribui, instiga e desenvolve no estudante de graduação características profissionais adequadas às necessidades da população.

Apesar da frágil relação academia-serviço servir como desestímulo, e da preocupação de ser identificado como não qualificado para a função, no exercício de um processo de trabalho desestruturado e fragmentado, os preceptores remanescentes ainda têm a esperança de solução aos entraves nessa relação.

"Hoje, alguns desses colegas são preceptores e recebem esses novos alunos e veem que a formação deles está mais completa, isso veio fortalecer muito esses dois anos de internato, dedicados, especificamente aos estágios. A prática dos estágios melhorou muito, melhorou muito essa formação médica."

As reflexões dos preceptores de Maceió confirmam e ampliam resultados de estudo anterior que identificou aspectos relevantes para a satisfação no exercício da preceptoria: capacitação pedagógica, reconhecimento e regulamentação da função do preceptor junto à universidade no processo ensino-aprendizagem, apoio para a aprendizagem de metodologias de ensino referenciadas nas correntes críticas da educação, e valorização da 
função do preceptor com ênfase na mobilização das capacidades individuais e sociais de transformação das práticas assistenciais e educacionais (Jesus \& Ribeiro, 2012).

\section{CONSIDERAÇÕES FINAIS}

Na compreensão dos preceptores entrevistados, a atenção básica tem contribuído para a formação dos discentes de medicina, bem como o exercício da preceptoria tem impulsionado o processo ensino-aprendizagem, provendo conhecimento dos atributos da APS e promovendo a autonomia, apesar da gestão ineficiente da IES para garantir direitos.

Os preceptores reconhecem a importância da atenção básica na formação profissional médica, em todas as etapas de formação, com ênfase na experiência prática durante o internato. Esses têm consciência que a presença do aluno tem contribuído para maior reflexão sobre o processo de trabalho. No entanto, a IES continua fragilizada, os preceptores percebem-se isolados, a comunicação academia-serviço é falha e insuficiente, não havendo planejamento conjunto. A indefinição de responsabilidades e seus respectivos papéis entre as partes envolvidas também tem contribuído para que os profissionais relatem a sensação de vazio, de não pertencimento no processo.

O método qualitativo com apoio no software iramuteq foi útil para definição de categorias e seleção de conteúdos discursivos para análise. O software acelerou os processos de análise e permitiu aos pesquisadores comparar os elementos selecionados com suas leituras subjetivas individuais e ampliar o foco da interpretação. Os limites ao método encontram-se na impossibilidade de apresentar para publicação todos os gráficos gerados, muito interessantes, e auxiliares à interpretação dos resultados.

\section{REFERÊNCIAS}

Barreto, V. H., Monteiro, R. O. S., Magalhães, G. S. G., Almeida, R. C. C., \& Souza, L. N. (2011). Papel do preceptor da atenção primária em saúde na formação da graduação e pós-graduação da Universidade Federal de Pernambuco: Um termo de referência. Revista Brasileira de Educação Médica, 35(4), 578-583.

Bentes, A., Leite, A. J. M., Montenegro, A. P. D. R., Paiva Junior, B. R., Medeiros, S. F. C., Gomes, J. M. A., Afonso, D. H. (2013, Outubro). Preceptor de residência médica: Funções, competência e desafios. A contribuição de quem valoriza porque percebe a importância: Nós mesmos! Cadernos da Associação Brasileira de Educação Médica, 9, 32-38.

Camargo, B., \& Justo, A.M. (2013). Iramuteq: Um software gratuito para análise de dados textuais. Temas em Psicologia, 21(2), 513-18.

Cavalcante, J. K., Soares, F. J. P., \& Correia, D. S. (2014). Desenvolvimento discente no estágio em estratégia saúde da família. Revista Brasileira de Educação Médica, 38(1), 15-24.

Cavalheiro, M. T. P., \& Guimarães, A. L. (2011, Dezembro). Formação para o SUS e os desafios da integração ensino serviço. Caderno do Fórum nacional de educação das profissões na área da saúde, 1, $19-27$.

Ceccim, R. B., \& Feuerwerker, L. C. M. (2004). Mudança na graduação dos profissionais de saúde sob o eixo da integralidade. Cadernos de saúde pública, 20(5), 1400-1410. 
Cyrino, E. G., Pinto, H. A., Oliveira, F. P., \& Figueiredo, A. M. (2015). O Programa Mais Médicos e a formação no e para o SUS: por que a mudança? Escola Anna Nery, 19(1), 5-10.

Decreto no 7.508 (2011, Junho). Regulamenta a Lei no 8.080, de 19 de setembro de 1990, para dispor sobre a organização do Sistema Único de Saúde - SUS, o planejamento da saúde, a assistência à saúde e a articulação interfederativa, e dá outras providências. Disponível em http://www.jusbrasil.com.br/legislacao/1028206/decreto-7508-11

Jesus, J. C. M., \& Ribeiro, V. M. B. (2012). Uma avaliação do processo de formação pedagógica de preceptores do internato médico. Revista Brasileira de Educação Médica, 36(2), 153-161.

Lei no 8.080. (1999, Setembro). Dispõe sobre as condições para a promoção, proteção e recuperação da saúde, a organização e o funcionamento dos serviços correspondentes e dá outras providências. Diário Oficial da União. Brasília, Seção 1.

Lima, P. A. B., \& Rozendo, C. A. (2015). Desafios e possibilidades no exercício da preceptoria do Pró-PETSaúde. Interface - Comunicação, saúde, educação, 19(1), 779-91.

Lins, T. S., Soares, F. J. P., \& Coelho, A. P. M. (2016). Avaliação dos atributos em atenção primária à saúde no estágio em saúde da família. Revista Brasileira de Educação Médica, 40(3), 355-363.

Ministério da Educação e Cultura. (2001, Novembro). Resolução CNE/CES no 4, de 07/2001. diretrizes curriculares nacionais do curso de graduação em medicina. Brasília, Brasil: Ministério da Educação e Cultura.

Ministério da Educação e Cultura. (2014, Junho). Resolução CNE/CES nํㅜ 3/2014. diretrizes curriculares nacionais do curso de graduação em medicina. Brasília, Brasil: Ministério da Educação e Cultura.

Ministério da Saúde. (2005). Pró -saúde: Programa nacional de reorientação da formação profissional em saúde. Brasília, Brasil: Ministério da Saúde.

Missaka, H., \& Ribeiro, V. M. B. (2011). Preceptoria na formação médica: Subsídios para integrar teoria e prática na formação profissional - o que dizem os trabalhos nos congressos brasileiros de educação médica 20072009. Revista Brasileira de Educação Médica, 35(3), 303-310.

Peduzzi, M., Norman, I. J., Germani, A. C. C. G., Silva, J. A. M., Souza, G. C. (2013). Educação interprofissional: Formação de profissionais de saúde para o trabalho em equipe com foco nos usuários. Revista da Escola de Enfermagem da USP, 47(4), 977-983.

Pinheiro, R., \& Luz, M. T. (2003). Práticas eficazes x modelos ideais: Ação e pensamento na construção da integralidade. In Construção da integralidade: cotidiano, saberes e práticas em saúde (pp. 1-25). Rio de Janeiro, Brasil: Associação Brasileira de Saúde Coletiva.

Portaria interministerial $\mathrm{n}$ - 1.127. (2015, Agosto). Institui as diretrizes para a celebração dos Contratos Organizativos de Ação Pública Ensino-Saúde (COAPES), para o fortalecimento da integração entre ensino, serviços e comunidade no âmbito do Sistema Único de Saúde (SUS).

Ratinaud, P. (2014). Iramuteq: Interface de R pour les Analyses Multidimensionnelles de Textes et de Questionnaires (Version 0.7 alpha 2) [Windows, GNU/Linux, Mac OS X]. Consulté à l'adresse http://www.iramuteq.org

Rodrigues, A. A., Juliano, I. A., Melo, M. L. C., Beck, C. L. C., \& Prestes, F. C. (2012). Processo de interação ensino, serviço e comunidade: a experiência de um PET-Saúde. Revista Brasileia de Educação Médica, 36(1), 184-192.

Sisson, M. C. (2009). Implantação de programas e redefinição de práticas profissionais. Revista Brasileira de Educação Médica, 33(1), 92-103.

Sousa, D. P. (2013). Preceptoria em saúde bucal na atenção básica no município de Goiânia sob a perspectiva do preceptor (Dissertação de mestrado, Universidade Federal de Goiás, Goiânia, Brasil).

Trajman, A., Assunção, N., Venturi, M., Tobias, D., Toschi, W., \& Brant, V. (2009). A preceptoria na rede básica da secretaria municipal de saúde do Rio de Janeiro: Opinião dos profissionais de saúde. Revista Brasileira de Educação Médica, 33(1), 24-32. 